\title{
Seiberg-Witten Monopole Equations And Riemann Surfaces
}

\author{
Cihan Saçlıoğlu ${ }^{1,2}$ \\ and \\ Serdar Nergiz ${ }^{1}$ \\ ${ }^{1}$ Physics Department, Bog̃aziçi University \\ 80815 Bebek-İstanbul, Turkey \\ and \\ ${ }^{2}$ Physics Department, TUBITAK \\ Marmara Research Center \\ Research Institute for Basic Sciences \\ 41470 Gebze, Turkey
}

\begin{abstract}
The twice-dimensionally reduced Seiberg-Witten monopole equations admit solutions depending on two real parameters $(b, c)$ and an arbitrary analytic function $f(z)$ determining a solution of Liouville's equation. The $U(1)$ and manifold curvature 2 -forms $F$ and $R_{2}^{1}$ are invariant under fractional $S L(2, \mathbb{R})$ transformations of $f(z)$. When $b=1 / 2$ and $c=0$ and $f(z)$ is the Fuchsian function uniformizing an algebraic function whose Riemann surface has genus $p \geq 2$, the solutions, now $S L(2, \mathbb{R})$ invariant, are the same surfaces accompanied by a $U(1)$ bundle of $c_{1}= \pm(p-1)$ and a 1-component constant spinor.
\end{abstract}




\section{Introduction:}

The study of the Donaldson invariants of 4-manifolds has become considerably simpler in Witten's [1] new approach based on weakly interacting $U(1)$ monopoles. Instead of self-dual Yang-Mills fields on the manifold, one now deals with a Weyl spinor $\psi$ and a $U(1)$ connection $A_{\mu}$, whose interaction is described by the Seiberg-Witten monopole equations (SWME)

$$
\not D{ }_{A} \psi=0
$$

and

$$
F_{\mu \nu}^{+}=-\frac{i}{4} \psi^{\dagger}\left[\gamma_{\mu}, \gamma_{\nu}\right] \psi
$$

where $F_{\mu \nu}^{+}$represents the self-dual part of $F_{\mu \nu}=\partial_{\mu} A_{\nu}-\partial_{\nu} A_{\mu}$.

An immediate consequence of (1) and (2), obtained by using the Weitzenbock formula, is that nontrivial and nonsingular solutions are possible only when the scalar curvature $R$ is negative in some regions of the manifold. Witten's vanishing theorem [1] , based on an integrated version of the same argument, establishes that $R \geq 0$ solutions are not only singular but also non- $L^{2}$. Nevertheless, such solutions may be worth studying if they are related to field configurations of physical interest. For example, Freund [2] has exhibited a once-dimensionally reduced solution in $\mathbb{R}^{4}$, also found earlier by Gürsey [3] in another setting, corresponding

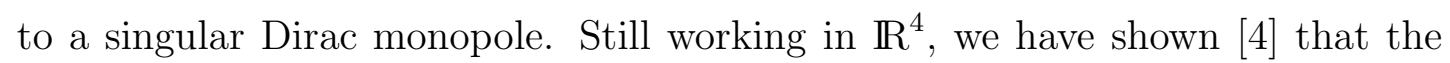
SWME allow singular multi-vortices and singular $\varphi^{4}$-kink solutions (the stan- 
dard non-singular versions are obtained by passing from $(++++)$ to $(++--)$ signature) when dimensionally reduced to $d=2$ and $d=1$, respectively.

In this paper we generalize our search for $d=2$ solutions by introducing a nonflat metric in two dimensions. The curvature can now assume negative values, and non-singular solutions become possible. As will be seen below, a subset of these solutions are nothing but Riemann surfaces of genus $p>1$ with Chern number $p-1$ ! These topological features are found to be invariant under a wide class of transformations on the metric and other fields. This is perhaps a reflection of the connection, pointed out by Olsen [0], between twice-dimensionally reduced SWME and certain $2 d$ topological field theories [6].

Let us briefly summarize the paper. In section 2, we write down the SWME for a product manifold $\mathcal{M}_{4}=\mathcal{M}_{2} \otimes S^{1} \otimes S^{1}$, where the radii of the circles have been taken to zero (of course, as long as we keep only the lowest modes, we could also consider $\mathcal{M}_{2} \otimes S^{2}$ ). We then specialize to a simple Ansatz which reduces the SWME to a covariant form of the Poisson-Boltzmann equation appearing in non-singular multivortex solutions given in [7] and [8]. In section 3 , we present a special set of solutions which depend on two parameters $b$ and $c$ and an arbitrary analytic function $g(z)$ characterizing a solution of Liouville's equation. In section 4 , we show that $b$ determines the sign of the curvature $R_{212}^{1}$ of $\mathcal{M}_{2}$ (and thus also its topology, through the Gauss-Bonnet formula), while changing $c$ only effects $U(1)$ gauge transformations on $\psi$ and $A_{\mu}$, simultaneously with 
a conformal transformation on the metric $h_{\alpha \beta}$ of $\mathcal{M}_{2}$. In section 5 , the same transformation property is seen to hold when $g(z)$ is subjected to the fractional version of $S U(1,1)$ transformations $\tilde{g}=(\alpha g+\beta) /(\bar{\beta} g+\bar{\alpha})$ with $\alpha \bar{\alpha}-\beta \bar{\beta}=1$. In section 6 , we study in greater detail the solutions that correspond to specific values of $b$ and $c$; the case $b=1$ is seen to revert to our earlier [4] singular multivortices in $\mathbb{R}^{4}$, while $b>1$ duly leads to singular $R \geq 0$ solutions. On the other hand, $b=1 / 2, c=0$ yields a constant negative curvature $\mathcal{M}_{2} ;$ according to a well-known theorem, one can obtain all genus $p \geq 2$ Riemann surfaces by dividing this $\mathcal{M}_{2}$ by appropriate discrete groups. This 'division' or tesellation of the hyperboloid by $4 p$-gons with geodesic edges corresponds to expressing $g(z)$ in terms the Fuchsian functions introduced by Poincaré. Remarkably, a recent analysis [9] of the Seiberg-Witten Ansatz [10] in its original physics context (quark confinement via a monopole condensate) reveals that the essence of the approach consists in identifying the function $\mathcal{F}^{\prime \prime}(a)$ governing the effective supersymmetric Lagrangian with the simplest Fuchsian function, which happens to be the inverse of the elliptic modular function! With the choice of a suitable Fuchsian function for $g(z), \mathcal{M}_{2}$ becomes a Riemann surface of Euler characteristic $\chi=2-2 p$ accompanied by a $U(1)$ bundle of Chern number (physically, the number of vortices) $c_{1}=p-1$ and a constant Weyl spinor. Section 7 ends the paper with concluding remarks concerning Riemann surfaces, Integrable Systems and the Seiberg-Witten equations . 


\section{Reduction of the SWME to $d=2$ :}

Any $\mathcal{M}_{2}$ metric $h_{\alpha \beta}$ can be written in the conformally flat form $h_{\alpha \beta}=e^{2 \phi} \delta_{\alpha \beta}$. After dimensional reduction, the basis 1-forms may be taken as

$$
e^{1}=e^{\phi} d x^{1}, \quad e^{2}=e^{\phi} d x^{2} \quad, \quad e^{3}=d x^{3} \quad, \quad e^{4}=d x^{4},
$$

where $\phi=\phi\left(x^{1}, x^{2}\right)$. The Cartan structure equations yield

$$
\omega_{2}^{1}=\left(\partial_{2} \phi\right) d x^{1}-\left(\partial_{1} \phi\right) d x^{2} .
$$

The curved-index $\gamma$-matrices are

$$
\gamma^{\mu}=\gamma^{a} E_{a}^{\mu}
$$

Here $E_{a}^{\mu} e_{\mu}^{b}=\delta_{a}^{b} ;$ thus

$$
E_{1}=e^{-\phi} \partial_{1}, \quad E_{2}=e^{-\phi} \partial_{2}, \quad E_{3}=\partial_{3} \quad, \quad E_{4}=\partial_{4} .
$$

We take the flat-space $\gamma$-matrices as

$$
\begin{aligned}
& \gamma^{1}=\tau_{1} \otimes \sigma_{1}=\left(\begin{array}{cc}
\mathbf{0} & \sigma_{1} \\
\sigma_{1} & \mathbf{0}
\end{array}\right), \quad \gamma^{2}=\tau_{1} \otimes \sigma_{2}=\left(\begin{array}{cc}
\mathbf{0} & \sigma_{2} \\
\sigma_{2} & \mathbf{0}
\end{array}\right) \\
& \gamma^{3}=\tau_{1} \otimes \sigma_{3}=\left(\begin{array}{cc}
\mathbf{0} & \sigma_{3} \\
\sigma_{3} & \mathbf{0}
\end{array}\right), \gamma^{4}=\tau_{2} \otimes \mathbb{1}=\left(\begin{array}{cc}
\mathbf{0} & -i \mathbb{1} \\
i \mathbb{1} & \mathbf{0}
\end{array}\right) \\
& \gamma^{5} \equiv \gamma^{4} \gamma^{1} \gamma^{2} \gamma^{3}=\left(\begin{array}{cc}
\mathbb{1} & \mathbf{0} \\
\mathbf{0} & -\mathbb{1}
\end{array}\right) .
\end{aligned}
$$

These act on the Weyl spinor $\psi^{T}=\left(\psi_{1}\left(x^{1}, x^{2}\right), \psi_{2}\left(x^{1}, x^{2}\right), 0,0\right)$. One of the SWME is the Dirac equation

$$
\not D_{A} \psi=0
$$


where

$$
\not D_{A}=\gamma^{a} E_{a}^{\mu}\left(\partial_{\mu}+i A_{\mu}+\frac{1}{8} \omega_{\mu}^{b c}\left[\gamma_{b}, \gamma_{c}\right]\right)
$$

Written explicitly, (9) becomes the pair of equations

$$
e^{\phi}\left(i A_{3}-A_{4}\right) \psi_{1}+\left(\partial_{1}-i \partial_{2}+i A_{1}+A_{2}+\frac{1}{2} \partial_{1} \phi-\frac{i}{2} \partial_{2} \phi\right) \psi_{2}=0
$$

and

$$
e^{\phi}\left(-i A_{3}-A_{4}\right) \psi_{2}+\left(\partial_{1}+i \partial_{2}+i A_{1}-A_{2}+\frac{1}{2} \partial_{1} \phi+\frac{i}{2} \partial_{2} \phi\right) \psi_{1}=0
$$

It is of course understood that $A_{\mu}=A_{\mu}\left(x^{1}, x^{2}\right)$ only. The remaining SWME are

$$
F_{\mu \nu}^{+}=-\frac{i}{4} \psi^{\dagger}\left[\gamma_{\mu}, \gamma_{\nu}\right] \psi
$$

where

$$
F_{\mu \nu}^{+}=\frac{1}{2}\left(F_{\mu \nu}+\frac{1}{2} \epsilon_{\mu \nu \alpha \beta} F^{\mu \nu}\right)
$$

and

$$
F_{\mu \nu}=\partial_{\mu} A_{\nu}-\partial_{\nu} A_{\mu}
$$

as usual. The three components in (11) yield

$$
\begin{gathered}
\partial_{1} A_{2}-\partial_{2} A_{1}=e^{2 \phi}\left(\left|\psi_{1}\right|^{2}-\left|\psi_{2}\right|^{2}\right), \\
\partial_{2} A_{3}+e^{-2 \phi} \partial_{1} A_{4}=e^{\phi}\left(\overline{\psi_{1}} \psi_{2}+\overline{\psi_{2}} \psi_{1}\right),
\end{gathered}
$$

and

$$
\partial_{2} A_{4}-e^{-2 \phi} \partial_{1} A_{3}=i e^{\phi}\left(\overline{\psi_{1}} \psi_{2}-\overline{\psi_{2}} \psi_{1}\right)
$$


The most general form of the SWME depending on two variables is represented by the set (10) and (14), involving 5 real $\left(A_{\mu}, \phi\right)$ and two complex $\left(\psi_{1}, \psi_{2}\right)$ functions. In order to obtain a solution, we will consider progressively more restricted Ansätze. We start by looking for solutions with $A_{3}=A_{4}=0$, which, by (14.b) and (14.c), implies that either $\psi_{1}=0$ or $\psi_{2}=0$. Let us begin with the case $\psi_{2}=0$. The SWME are now reduced to

$$
\left(\partial_{1}+i \partial_{2}+i A_{1}-A_{2}+\frac{1}{2} \partial_{1} \phi+\frac{i}{2} \partial_{2} \phi\right) \psi_{1}=0
$$

and

$$
\partial_{1} A_{2}-\partial_{2} A_{1}=e^{2 \phi}\left|\psi_{1}\right|^{2}
$$

It is useful to write

$$
\psi_{1}=a \exp \left(\omega_{x}+i \omega_{y}\right),
$$

where $\omega_{x}$ and $\omega_{y}$ are real functions and $a$ is a positive constant with the dimensions of inverse length. Note that because the vacuum expectation value of the Higgs field from the original twisted supersymmetric Yang-Mills theory has [11] been incorporated into the equations, the spinor field dimension is no longer the usual $[L]^{-3 / 2}$. Dividing (15) by $\psi_{1}$, applying $\partial_{1}-i \partial_{2}$ on the result, separating real and imaginary parts and using (16) lead to the pair of equations

$$
\left(\partial_{1} \partial_{1}+\partial_{2} \partial_{2}\right)\left(\omega_{x}+\phi / 2\right)=a^{2} \exp \left(2 \omega_{x}+2 \phi\right)
$$


and

$$
\left(\partial_{1} \partial_{1}+\partial_{2} \partial_{2}\right) \omega_{y}=-\left(\partial_{1} A_{1}+\partial_{2} A_{2}\right)
$$

Making use of

$$
R_{2}^{1}=d \omega_{2}^{1}=-\left(\partial_{1} \partial_{1} \phi+\partial_{2} \partial_{2} \phi\right) e^{-2 \phi} e^{1} \wedge e^{2}
$$

and

$$
\sqrt{\operatorname{det}\left(h_{\alpha \beta}\right)}=e^{2 \phi}
$$

(18) can be written in the form

$$
\left(\omega_{x}\right)_{; \mu}^{; \mu}-\frac{1}{2} R=a^{2} e^{2 \omega_{x}}
$$

where $R$ is the scalar curvature.

(22) represents a covariant generalization of some well-known non-linear field equations in two dimensions. For instance, when the metric is flat $(\phi=0),(22)$ reduces to the Liouville equation, whose solutions are singular along a closed curve in the $x^{1} x^{2}$-plane; this is the case already examined in [4]. For constant negative curvature, (22) becomes the covariant form of the Poisson-Boltzmann equation which arises in a number of seemingly unrelated contexts, e.g., in the DebyeHückel theory [8] of electrolytic solutions, as well as in the parametrization of noninteracting two-dimensional multivortex solutions without singularities [7]. Since the constant term in the Poisson-Bolzmann equation helps cure the Liouville singularities in the multivortex problem, one may also expect (22) to yield nonsingular solutions for appropriate negative curvature metrics $h_{\alpha \beta}$. 


\section{A 2-parameter class of special solutions:}

Obtaining the general solution of (22) for a given metric $h_{\alpha \beta}$ is a highly nontrivial task. However, a special class of solutions is readily available. Let us first introduce a parameter $b$ and write

$$
\omega_{x}+\phi / 2=b\left(\omega_{x}+\phi\right)+\left[(1-b) \omega_{x}+(1-2 b) \phi / 2\right]
$$

and then impose

$$
\left(\partial_{1} \partial_{1}+\partial_{2} \partial_{2}\right)\left[(1-b) \omega_{x}+(1-2 b) \phi / 2\right]=0
$$

Next, define the dimensionless complex coordinates

$$
z \equiv \frac{a}{\sqrt{b}}\left(x^{1}+i x^{2}\right), \quad \bar{z} \equiv \frac{a}{\sqrt{b}}\left(x^{1}-i x^{2}\right)
$$

in terms of which (18) (which is another form of (22)) becomes

$$
4 \partial_{z} \partial_{\bar{z}}\left(\omega_{x}+\phi\right)=e^{2\left(\omega_{x}+\phi\right)}
$$

since (24) holds. Thus we end up with the Liouville equation for the combination $\left(\omega_{x}+\phi\right)$, in contrast to the Liouville equation satisfied by $\omega_{x}$ alone in the $\mathbb{R}^{4}$ case. The solution of (26) is due to Liouville [12]; it is given by

$$
\omega_{x}+\phi=\frac{1}{2} \ln \left(4 g^{\prime} \bar{g}^{\prime}\right)-\ln (1-g \bar{g})
$$

where $g(z)$ denotes an arbitrary analytic function, $\bar{g}$ its antianalytic complex conjugate and $g^{\prime}=d g / d z$. One satisfies (24) automatically by taking

$$
(1-b) \omega_{x}+(1-2 b) \phi / 2=c \ln \left(4 g^{\prime} \bar{g}^{\prime}\right)^{1 / 2}
$$


as $\partial_{z} \partial_{\bar{z}}$ annihilates the right-hand side of (28); $c$ is an additional arbitrary parameter. Now solving for $\phi$ and $\omega_{x}$ from (28) and (27), we find

$$
\omega_{x}=[2(b+c)-1] \ln \sqrt{\left(4 g^{\prime} \bar{g}^{\prime}\right)}+(1-2 b) \ln (1-g \bar{g})
$$

and

$$
\phi=2[1-(b+c)] \ln \sqrt{\left(4 g^{\prime} \bar{g}^{\prime}\right)}-2(1-b) \ln (1-g \bar{g}) .
$$

From $\left|\psi_{1}\right|=a \exp \omega_{x}$, we have

$$
\left|\psi_{1}\right|=a \frac{\left(4 g^{\prime} \bar{g}^{\prime}\right)^{b+c-1 / 2}}{(1-g \bar{g})^{2 b-1}}
$$

It is then natural to take

$$
\psi_{1}=a e^{\omega_{x}+i \omega_{y}}=a \frac{\left(2 g^{\prime}\right)^{2 b+2 c-1}}{(1-g \bar{g})^{2 b-1}} .
$$

which means we have chosen

$$
\omega_{y}=i(b+c-1 / 2) \ln \left(\bar{g}^{\prime} / g^{\prime}\right) .
$$

Solving for the $U(1)$ connection from (15), we obtain

$$
A_{1}=\frac{i a}{2 \sqrt{b}}\left\{[1-(b+c)]\left(\frac{\bar{g}^{\prime \prime}}{\bar{g}^{\prime}}-\frac{g^{\prime \prime}}{g^{\prime}}\right)+2 b \frac{\left(g \bar{g}^{\prime}-g^{\prime} \bar{g}\right)}{(1-g \bar{g})}\right\}
$$

and

$$
A_{2}=\frac{a}{2 \sqrt{b}}\left\{[1-(b+c)]\left(\frac{\bar{g}^{\prime \prime}}{\bar{g}^{\prime}}+\frac{g^{\prime \prime}}{g^{\prime}}\right)+2 b \frac{\left(g \bar{g}^{\prime}+g^{\prime} \bar{g}\right)}{(1-g \bar{g})}\right\} .
$$

With these $\left(A_{1}, A_{2}\right)$ and $\omega_{y}$, equation (19) is seen to hold with both sides vanishing. One may now go back and check that (30), (32) and (34) solve the original SWME. 
It is useful to express the $U(1)$ connection as a 1 -form. This gives

$$
A=\frac{i}{2}[1-(b+c)] d \ln \left(\bar{g}^{\prime} / g^{\prime}\right)+i b \frac{(g d \bar{g}-\bar{g} d g)}{(1-g \bar{g})} .
$$

The spin-connection 1-form is

$$
\omega_{2}^{1}=i[1-(b+c)] d \ln \left(g^{\prime} / \bar{g}^{\prime}\right)+2 i(1-b) \frac{(\bar{g} d g-g d \bar{g})}{(1-g \bar{g})} .
$$

One may define complex basis 1 -forms via

$$
e^{z} \equiv e^{1}+i e^{2}=\frac{\sqrt{b}}{a} d z \frac{\left(4 g^{\prime} \bar{g}^{\prime}\right)^{1-(b+c)}}{(1-g \bar{g})^{2-2 b}}
$$

and

$$
e^{\bar{z}} \equiv e^{1}-i e^{2}=\frac{\sqrt{b}}{a} d \bar{z} \frac{\left(4 g^{\prime} \bar{g}^{\prime}\right)^{1-(b+c)}}{(1-g \bar{g})^{2-2 b}}
$$

The curvature 2-form for the $U(1)$ connection

$$
F=d A=2 i b \frac{d g \wedge d \bar{g}}{(1-g \bar{g})^{2}}
$$

and the manifold

$$
R_{2}^{1}=d \omega_{2}^{1}=-4 i(1-b) \frac{d g \wedge d \bar{g}}{(1-g \bar{g})^{2}}
$$

are seen to be proportional to each other. Stripping away the basis 1-forms, we have

$$
R_{212}^{1}=-2 a^{2}\left(\frac{1-b}{b}\right) \frac{\left(4 g^{\prime} \bar{g}^{\prime}\right)^{2(b+c)-1}}{(1-g \bar{g})^{4 b-2}}
$$

and

$$
F_{12}=\frac{4 a^{2} g^{\prime} \bar{g}^{\prime}}{(1-g \bar{g})^{2}}
$$


for the Riemann and $U(1)$ field strength tensors, respectively. In two dimensions $R_{212}^{1}$ of course coincides with the scalar curvature $R$. The equation (41) shows that

$\begin{array}{llll}\text { (i) } & R<0 & \text { for } & 1>b>0 \\ \text { (ii) } & R>0 & \text { for } & b>1 \\ \text { (iii) } & R=0 & \text { for } & b=1 .\end{array}$

One might think (41) also allows $R>0$ when $b<0$, but a glance at (25) reveals that negative values of $b$ lead to $z$ and $\bar{z}$ being multiplied by $\sqrt{-1}$, resulting in a signature change from $(++++)$ to $(--++)$. This would also require changes in the Diracology; thus our framework restricts us to $b>0$ as (25) shows $b=0$ is also to be excluded.

The three possibilities in (43) may remind the reader of a fundamental theorem in two dimensional geometry and topology which states that all Riemann surfaces can be derived from the sphere $S^{2}$, the complex plane $\mathbb{C}$ or the constant negative curvature hyperboloid $H^{2}$; in particular, the genus $p=1$ torus and $p \geq 2$ surfaces are obtained by dividing $\mathbb{C}$ and $H^{2}$ by appropriate discrete groups. Conformal transformations can be used to generate surfaces homeomorphic to the above. We will indeed see later that the genus $p>1$ surfaces emerge as a specific subset of our solutions for particular choices of the analytic function $g(z)$ and the parameters $b$ and $c$. 


\section{The significance of the parameters $b$ and $c$ :}

Using the Gauss-Bonnet theorem expression

$$
\frac{1}{2 \pi} \int R_{2}^{1}=\chi
$$

for the Euler characteristic $\chi$ and the definition of the first Chern number

$$
\frac{1}{2 \pi} \int F=c_{1}
$$

together with (39) and (40), we see that the topological invariants depend on $b$ but not on $c$. One therefore expects that the transformation

$$
c \rightarrow c+\delta c
$$

can at most result in a combination of a $U(1)$ gauge transformation and a con-

formal change of the metric $h_{\alpha \beta}$. Applying (46) on (32), (35), (36) and (41) one indeed finds

$$
\begin{gathered}
A \rightarrow A-\frac{i}{2} \delta c d \ln \left(\bar{g}^{\prime} / g^{\prime}\right), \\
\omega_{2}^{1} \rightarrow \omega_{2}^{1}+i \delta c d \ln \left(\bar{g}^{\prime} / g^{\prime}\right), \\
R_{212}^{1} \rightarrow\left(4 \bar{g}^{\prime} g^{\prime}\right)^{2 \delta c} R_{212}^{1}, \\
\operatorname{det}\left(h_{\alpha \beta}\right)^{1 / 2} \equiv h^{1 / 2}=e^{2 \phi} \rightarrow\left(4 \bar{g}^{\prime} g^{\prime}\right)^{-2 \delta c} e^{2 \phi},
\end{gathered}
$$

and

$$
\psi_{1} \rightarrow\left(2 g^{\prime}\right)^{2 \delta c} \psi_{1}
$$

At first sight it might appear that (51) is not the anticipated $U(1)$ transformation for a spinor wavefunction until one realizes, on both physical and mathematical 
grounds, that in the presence of a non-flat $\mathcal{M}_{2}$ metric $h_{\alpha \beta}$, the usual $U(1)$ transformation must apply to the spinor density $\psi_{1} h^{1 / 4}$, which does transform in the expected way

$$
\psi_{1} h^{1 / 4} \rightarrow\left(g^{\prime} / \bar{g}^{\prime}\right)^{\delta c} \psi_{1} h^{1 / 4}
$$

\section{5. $S U(1,1)$ symmetry of the solutions :}

The reader may have noticed that there is a bigger symmetry group, beyond the 1-parameter group noted in section 4 , that leaves the 2 -form $d g \wedge d \bar{g} /(1-g \bar{g})^{2}$ (and hence the topology ) invariant. This consists of the well-known fractional transformations

$$
\tilde{g}=\frac{\alpha g+\beta}{\bar{\beta} g+\bar{\alpha}} \text { with }|\alpha|^{2}-|\beta|^{2}=1 .
$$

Thus $\left(\begin{array}{cc}\alpha & \beta \\ \bar{\beta} & \bar{\alpha}\end{array}\right) \in S U(1,1)$ and $|g| \leq 1$ is mapped into $|\tilde{g}| \leq 1$. The effect of (53) on the fields is given by

$$
\begin{gathered}
A \rightarrow A-i(2 b+c-1) d \ln \left(\frac{\bar{\beta} g+\bar{\alpha}}{\beta \bar{g}+\alpha}\right), \\
\omega_{2}^{1} \rightarrow \omega_{2}^{1}+2 i c d \ln \left(\frac{\bar{\beta} g+\bar{\alpha}}{\beta \bar{g}+\alpha}\right), \\
\psi_{1} \rightarrow \psi_{1} \frac{|\bar{\beta} g+\bar{\alpha}|^{(4 b-2)}}{(\bar{\beta} g+\bar{\alpha})^{(4 b+4 c-2)}}, \\
\psi_{1} h^{1 / 4} \rightarrow \psi_{1} h^{1 / 4}\left\{\frac{\beta \bar{g}+\alpha}{\bar{\beta} g+\bar{\alpha}}\right\}^{2 b+2 c-1}, \\
h^{1 / 2}=e^{2 \phi} \rightarrow|\bar{\beta} g+\bar{\alpha}|^{8 c} e^{2 \phi},
\end{gathered}
$$

and

$$
R_{212}^{1} \rightarrow|\bar{\beta} g+\bar{\alpha}|^{-8 c} R_{212}^{1}
$$


We note again the simultaneous occurence of $U(1)$ gauge transformations on $A$ and $\psi$, together with conformal transformations on $h_{\alpha \beta}, \omega_{2}^{1}$ and $R_{212}^{1}$. (47)-(52) and (54)-(59) are reminiscent of Hermann Weyl's early but mistaken attempt to obtain the form of the electromagnetic coupling from a requirement of local scale invariance; here Weyl's original 'Eichinvarianz' and the local $U(1)$ symmetry are actually linked!

The above fractional $S U(1,1)$ transformations on $g(z)$ are isomorphic to $S L(2, \mathbb{R})$ fractional transformations on the analytic function $f(z)$ defined by

$$
g(z)=\frac{f(z)-i}{f(z)+i}
$$

The relation (60) and its inverse map the region $|g| \leq 1$ to the upper half $f$-plane $\operatorname{Im} f \geq 0$ and vice versa. The counterpart of (53) is now

$$
\tilde{f}=\frac{A f+B}{C f+D}
$$

where, with

$$
\alpha \equiv p+i q, \quad \beta \equiv r+i s, \quad p, q, r, s \in R
$$

one has

$$
p=\frac{1}{2}(A+D), \quad q=\frac{1}{2}(B-C), \quad r=\frac{1}{2}(A-D), \quad s=\frac{-1}{2}(B-C)
$$

and

$$
|\alpha|^{2}-|\beta|^{2}=A D-B C=1 .
$$


The function $f$ is more convenient to work with than the function $g$ when one wishes to focus on the modular subgroup $S L(2, \mathbb{Z})$ of $S L(2, \mathbb{R})$.

\section{Special values of $b$ and $c$ and Riemann surfaces :}

Having seen that $c$ has no topological significance, we can set $c=0$ and concentrate on $b$, which must be greater than zero (recall the discussion at the end of section 3 and equation (25)). It is easy to see that $b$ can only assume a restricted set of values when $c=0$. Let us put $g=|g| e^{i \gamma}$ and take $\alpha=0$,

$\beta=1$. Although now $|\alpha|^{2}-|\beta|^{2}=-1$, this is still an admissible $U(1)$ gauge transformation under which (57) becomes

$$
\psi_{1} h^{1 / 4} \rightarrow \psi_{1} h^{1 / 4} e^{-2 i \gamma(2 b-1)}
$$

Demanding single valuedness of $\psi_{1} h^{1 / 4}$ under $\gamma \rightarrow \gamma+2 \pi$, we get

$$
b=\frac{n}{4}+\frac{1}{2}, n \in \mathbb{Z},
$$

the same conclusion also follows from (32).

We thus have the three cases below, where the first two are necessarily singular due to the Weitzenbock formula:

(i) $b>1(n \geq 3)$ : These solutions are homeomorphic to the sphere $\left(R_{212}^{1} \geq 0\right)$ except for a line of singularities along the curve $|g|=1$; we consider them no further.

(ii) $b=1(n=2)$ : The curvature $R_{212}^{1}$ now vanishes, but the $U(1)$ curvature 2-form $F$ does not. We have shown [包] earlier that elliptic or hyperelliptic curves 
of the form $g^{2}(z)=\left(z-a_{1}\right)\left(z-a_{2}\right) \ldots\left(z-a_{k}\right)$ lead to solutions with $k$ singular vortices centered at the locations $a_{1}, a_{2}, \ldots, a_{k}$ on the complex plane $\mathbb{C}$. Alternatively, one may [4] choose $g^{2}(z)=\sigma(z)$, where $\sigma$ is Weierstrass's quasi-doubly periodic function defined by $\sigma^{\prime} / \sigma \equiv \zeta(z) \equiv-\int \wp(z) d z$. As this amounts to dividing $\mathbf{C}$ by the lattice $\Gamma$ of points $\omega=n_{1} \omega_{1}+n_{2} \omega_{2}$, one ends up with a genus $p=1$ solution with one singular vortex per cell.

(iii) $1>b>0(n=-1,0,1):$ We now have $b=1 / 4,1 / 2$ and $3 / 4$. Pursuing the relation between our solutions and the classification of Riemann surfaces according to genus, we see the value $b=1 / 2$ assigns $\mathcal{M}_{2}$ the Poincaré metric

$$
d s^{2}=\frac{d g d \bar{g}}{(1-g \bar{g})^{2}}
$$

corrresponding to constant negative curvature $R_{212}^{1}=-2 a^{2}$. The $S U(1,1)$ symmetry discussed in section 5 is now an isometry of the metric (67). The Klein form of (67), obtained by replacing $g(z)$ by $f(z)$ via $(60)$, leads to the well-known metric

$$
d s^{2}=\frac{d f d \bar{f}}{(\operatorname{Im} f)^{2}}
$$

on the upper-half-plane $\mathbb{C}_{+}$. In the terminology of [9], $p \geq 2$ Riemann surfaces are obtained by dividing $\mathbb{C}_{+}$by $S L(2, \mathbb{Z})$ into $4 p$-gons $D_{p}$ with geodesic sides (arcs of circles of finite or infinite radius, intersecting $\operatorname{Im} f=0$ at right angles) ; the sides are of course identified according to the standard prescription given in, say, [13]. The identification is accomplished by choosing an $f_{p}(z)$ which maps $\mathbb{C}_{+}$to 
$D_{p}$; this means $f_{p}(z)$ should be an appropriate member of the family of Fuchsian functions [14] introduced by Poincaré. More precisely, $f_{p}(z)$ is a Fuchsian function of the first kind by which an algebraic function, whose Riemann surface has genus $p$, is uniformized [15]. Thus with $b=1 / 2, c=0, g(z)=\left(f_{p}(z)-i\right) /\left(f_{p}(z)+i\right)$, a compact, closed Riemann surface of genus $p \geq 2$ supporting $p-1$ non-singular 'magnetic vortices' and a constant Weyl spinor solves the SWME.

Turning to the $S U(1,1)$ transformation (54)-(59) or their $S L(2, \mathbb{R})$ versions based on (61), we see that neither $U(1)$ gauge transformations nor conformal changes in the metric are allowed when the choice $b=1 / 2, c=0$ is made; all the fields are now simply invariant! This fits in perfectly with the above solution based on $f_{p}(z)$ : the constant negative curvature hyperboloid (or, equivalently, the upper half-plane $\mathbb{C}_{+}$) has been tesellated by $4 p$-gons $D_{p}$ which transform into each other under the $S L(2, \mathbb{Z})$ subgroup of $S L(2, \mathbb{R})$; the invariance of the fields under the same transformations ensures that all the $D_{p}$ are completely equivalent.

For completeness, we summarize below some facts concerning $f_{p}(z)$. Taking the $4 p$ vertices of the polygon $D_{p}$ at the points $z=a_{\nu}(\nu=1, \ldots, 4 p)$ on the real axis, $f_{p}(z)$ is given by (in the notation of [9])

$$
f_{p}(z)=\frac{u_{1}(z)}{u_{2}(z)},
$$

where $u_{1}(z)$ and $u_{2}(z)$ are the two linearly independent solutions of

$$
u^{\prime \prime}(z)+\left[\frac{1}{4} \sum_{\nu=1}^{4 p} \frac{1-\alpha_{\nu}^{2}}{\left(z-\alpha_{\nu}\right)^{2}}+\frac{1}{2} \sum_{\nu=1}^{4 p} \frac{\beta_{\nu}}{z-\alpha_{\nu}}\right] u(z)=0 .
$$


In $(\sqrt{70}), \pi \alpha_{\nu}$ is the angle at vertex $\nu$ and the real constants $\beta_{\nu}$ satisfy

$$
\sum_{\nu=1}^{4 p} \beta_{\nu}=0, \sum_{\nu=1}^{4 p}\left(2 \alpha_{\nu} \beta_{\nu}+1-\alpha_{\nu}^{2}\right)=0, \sum_{\nu=1}^{4 p}\left[\beta_{\nu} \alpha_{\nu}^{2}+\alpha_{\nu}\left(1-\alpha_{\nu}^{2}\right)\right]=0
$$

The chief difficulty in obtaining a power-series solution of (70) lies in relating the $4 p-3$ constants $\beta_{\nu}$ to the precise geometry of $D_{p}$.

Turning finally to the cases $b=1 / 4(n=-1)$ and $3 / 4(n=+1)$, we see that (41) indicates the curvature $R_{212}^{1}$ can have singularities or zeroes depending on the choice of $g(z)$. There is no obvious geometrical or topological interpretation for these solutions; we consider them no further.

\section{Concluding remarks :}

The reader may have wondered what kind of solutions would have followed had we made the choice $\left(\psi_{1}=0, \psi_{2} \neq 0\right)$ just before equation (15). It is not difficult to repeat the subsequent steps and see that this only results in $A_{\mu} \rightarrow-A_{\mu}$, $F_{12} \rightarrow-F_{12}$ and $\left(\psi_{1}(g, \bar{g}), 0\right) \rightarrow\left(0, \psi_{2}(\bar{g}, g)\right)$; everything else remains unchanged. Recalling the components $\psi_{1}$ and $\psi_{2}$ of a Weyl spinor correspond to particle and antiparticle states of the same chirality, we see that the new solution is simply the charge conjugate of the original one. Note that there are no solutions where some of the vortices are 'up' and others are 'down'.

The examples presented so far certainly do not exhaust the set of $2 d$ SWME solutions. First of all, even within our narrow Ansatz, there is initially no restriction on $h_{\alpha \beta}$ since the SWME do not constrain the form of the metric through, 
say, Einstein's equation in General Relativity. Thus one could try to solve (18) for an arbitrarily assigned conformal function $\phi$. One could also consider a more general Ansatz with $A_{3}$ and/or $A_{4}$ non-zero, with or without a 'warp factor' $\mu\left(x_{1}, x_{2}\right)$ modifying the basis one forms $e^{3}$ and $e^{4}$ from their simple form in (3) to $e^{3}=\mu d x^{3}, e^{4}=\mu d x^{4}$. There are reasons to believe that these more general versions of the twice-dimensionally reduced SWME will be found to be related to $2 d$ integrable systems other than those involving the Liouville equation. For example the relation of $N=2$ supersymmetry gauge theory to integrable systems has been pointed out in [16], [17] and [18]; the last reference especially emphasizes the Riemann surface structure and Toda dynamics (the simplest example of which is the Liouville equation!) underlying reference [10]. Starting from the integrable systems end of the connection, the non-linearization procedure in [19], [20] and [21] exhibits a formal similarity to equation (2), while the dimensionally-reduced form of equation (11) may be regarded as the counterpart of the Lax pair of the equations for $\psi_{t}$ and $\psi_{x}[22]$.

Finally, we would like to draw attention to a number of mathematical features shared by the Seiberg-Witten model, 10 exhibiting quark confinement through monopole condensation and the analysis presented here. In [10] the function $\mathcal{F}(a)$, which determines the local part of the $N=2$ SUSY effective Lagrangian, has a second derivative $\mathcal{F}^{\prime \prime}(a)$ which coincides with our $f_{1 / 2}$. Of course, formally putting $p=1 / 2$ in (69)-(71) merely provides a definition of the inverse of the 
elliptic modular function and is not meant to imply that we are dealing with a surface of fractional genus. In fact, the analysis in [10] is based on a genus one elliptic curve. Among our solutions, on the other hand, the non-singular ones of particular interest due to their topologically non-trivial properties are those that are closely related to higher genus hyperelliptic cases. These higher genus Riemann surfaces also appear in the context of $N=2$ SUSY Yang-Mills theory when gauge groups beyond $S U(2)$ are considered while our work makes no explicit reference to the original unbroken non-abelian gauge group. There also seems to be a parallelism between the function $f(z)$ in our work and the parameter $\tau=\theta / 2 \pi+4 \pi i / e^{2}$ in that both undergo $S L(2, \mathbb{Z})$ projective transformations. The elucidation of the deeper reasons behind these mathematical coincidences requires further study.

\section{Acknowledgements}

We have benefited greatly from discussions with M. Ark, T. Dereli, E. Ferapontov, S. Finashin, H. Gümral, R. Güven, A. Klyachko and Y. Nutku.

\section{References}

[1] E. Witten, Math. Res. Lett. 1, 769 (1994).

[2] P. G. O. Freund, J. Math. Phys. 36 , 2673 (1995).

[3] F. Gürsey, in Gauge Theories and Modern Field Theory, edited by R. Arnowitt and P. Nath (MIT, Cambridge, 1976), p.369. 
[4] S. Nergiz and C. Saçlıoglu, J. Math. Phys. 37 , 3753 (1996).

[5] K. Olsen, Mod. Phys. Lett. A 11, 1777 (1996).

[6] D. Birmingham, M. Rakowski and G. Thompson, Nucl. Phys. B 315, 577 (1989); G. Chapline and B. Grosman, Phys. Lett. B 223 , 336 (1989).

[7] A. Jaffe and C. Taubes, Vortices and Monopoles, Birkhaüser, (1980).

[8] M. Lohe, Phys. Lett. B 70,325 (1977).

[9] R. Flume, L O'Raifeartaigh and I. Sachs, hep-th/9611118.

[10] N. Seiberg and E. Witten, Nucl. Phys. B 426, 19 (1994).

[11] E. Witten, J. Math. Phys. 35, 5101 (1994).

[12] J. Liouville, J. Math. Appl. 18, 71 (1853).

[13] B. A. Dubrovin, A. T. Fomenko, and S. P. Novikov, Modern Geometry Vol. II , Springer-Verlag, NY (1985).

[14] Z. Nehari, Conformal Mapping, Dover, NY (1952).

[15] L. R. Ford, Automorphic Functions, Chelsea, NY (1951).

[16] A. Gorsky, I. Krichever, A. Marshakov, A. Mironov and A. Morozov, Phys. Lett. B355, 466 (1995).

[17] R. Donagi and E. Witten, hep-th/9510101, IASSNS-HEP-95-78. 
[18] E. Martinec and N. Warner, Nucl. Phys. B459, 97 (1996).

[19] Z. Qiao, J. Math. Phys. 34, 3110 (1993).

[20] Y. Zeng, Physica D73, 171 (1994).

[21] W. X. Ma, Q. Ding, W. G. Zhang and B. Q. Lu, Nuovo Cimento 111B, 1135 (1996).

[22] E. Ferapontov, private communication. 